\title{
A PERCEPÇÃO DA GESTANTE NA ATUAÇÃO DO ENFERMEIRO - FORTALECENDO O VÍNCULO MÃE-BEBÊ
}

\author{
The perception of pregnant women in the work of nurses - strengthening the mother- \\ baby bond
}

\author{
Marília Antunes de Abreu Moura \\ Curso de Enfermagem. Facer - Faculdade de Ceres, Ceres - GO, Brasil. \\ marilia_antunesam@hotmail.com \\ Michelle Katrine Costa Silva \\ Curso de Enfermagem. Facer - Faculdade de Ceres, Ceres - GO, Brasil. \\ michellekatrine@hotmail.com
}

\author{
Viviane Rodrigues Tavares \\ Mestre em Enfermagem \\ Docente da Faculdade de Ceres, Ceres - GO. Brasil. \\ viavevivi@hotmail.com
}

\section{Endereço para correspondência:}

Av. Brasil, S/N, Qd. 13 Morada Verde - GO, Fone: (62) 3323-1040,

E-mail: vianevivi@hotmail.com

RESUMO: Introdução: As ações de promoção, prevenção e assistência a gestante e ao recémnascido (RN) são fundamentais, pois, visão facultar o vinculo entre mãe e filho. Neste trabalho será relevante avaliarmos a percepção das gestantes ao reconhecer a importância da atuação dos enfermeiros frente aos cuidados prestados, fortalecendo o vinculo entre mãe-bebê. Objetivo: Identificar na literatura a percepção da gestante frente a atuação do enfermeiro nos primeiros cuidados com RN. Metodologia: Trata-se de uma revisão integrativa, de aspecto quantitativo. A coleta foi realizada através da base de dados online Scielo e Biblioteca Virtual em Saúde, entre o período de setembro a outubro de 2014, a amostra foi composta por 12 artigos científicos. Resultados e Discussão: Os artigos foram publicados em periódicos em diferentes áreas temáticas. Os descritores mais empregados pelos autores foram parto, relações afetivas, cuidados de enfermagem, pré-natal e maternidade. Na analise dos artigos foram encontrados sentimentos positivos e negativos das mulheres em relação mãe-filho e percepção das mulheres em relação aos profissionais de enfermagem. Segundo Piccinini (2012), no pré-natal o enfermeiro como cuidador é responsável por suprir as necessidades emocionais da mãe, alem da saúde física da gestante e do bebê antes do nascimento ficando evidente que os primeiros contatos propiciam o reconhecimento entre mãe e filho. Conclusão: A realização deste estudo possibilitou pesquisar, compreender e identificar o significado da contribuição da equipe de enfermagem para a formação do vinculo entre mãe e filho.

Palavras-chave: Percepção das gestantes. Cuidados de enfermagem. Vinculo entre mãe e filho. Recém-nascido.

ABSTRACT: Introduction: The actions of promotion, prevention and assistance to pregnant women and newborn (RN) are fundamental, because they intend to promote the bond between mother and son. In this work it is going to be important to evaluate the perception of pregnant women, once they recognize the importance of the nurse's work in relation to the care given, strengthening the bond between a mother-baby. Objective: Identify in literature the perception of pregnant woman in face of 
the nurse`s approach to the first care aid with the newborn. Methodology: It is an integrative review of quantitative aspect. The information was collected through the online database Scielo and Virtual Health Library, between the period from September to October 2014, the sample was composed of 12 scientific articles. Results and discussion: The articles were published in journals in different thematic areas. The descriptors most employed by the authors were calving, affective relations, nursing care, pre-natal and maternity. On the article's analysis were found both, positive and negative feelings from women in relation to the mother-child relationship, as well as, to their perception towards the nursing professionals. According to Piccinini (2012), in the prenatal the nursing professionals are responsible for supplying the emotional needs of the mother, in addition to the physical health of the pregnant woman's and the baby before birth evidencing that the first contacts provide the recognition between mother and son. Conclusion: Through this study was possible research, understand and identify the significance of the contribution provided by the nursing staff for the formation of a bond between mother and son.

Keywords: Perception of pregnant women. Nursing care. Bond between mother and son. Newborn. 


\section{INTRODUÇÃO}

2

Os primeiros cuidados com o recém-nascido (RN) após o parto são primordiais para a habituação do bebê. O RN passa por mudança de fase do meio intra para o extrauterino, adaptando gradativamente ao meio externo, passando por diversas mudanças como momentos sensíveis, predecessor de apego, sendo uma oportunidade em que a mãe será sensibilizada pelo bebê (CRUZ et al., 2007).

O vínculo entre mãe e recém-nascido é fundamental no período do nascimento e tem maior influência nessa fase do que nas próximas. Observado desde o período de gestação ao puerpério, é nesse intervalo em que a mulher enfrenta mudanças físicas e emocionais. Assim, para que o vínculo ocorra é necessário que a mãe esteja apta a ter uma vivência referente a gestação e ao puerpério (BORSA, 2007).

O pré-natal é o momento importante de acompanhamento da gestante durante esta fase inicial, sendo realizadas ações de forma individualizada buscando melhor qualidade na atenção á saúde da mulher. Para uma assistência qualificada deve-se compreender o que as gestantes pensam em relação ao pré-natal, desenvolver acolhimento, formar vinculo com elas e oferecer informações de modo que elas aprendam sobre o mesmo (BARRETO et al., 2013).

$\mathrm{Na}$ assistência ao pré-natal a equipe é responsável por suprir as necessidades emocionais da mãe, além da saúde física da gestante e do bebê. Dessa forma as percepções e sentimentos das gestantes se forem positivas em relação ao pré-natal, pode-se reduzir as preocupações sobre a própria saúde e do bebê, fortalecendo o vínculo mãe e filho (PICCININI et al., 2012).

Durante a hospitalização a mãe e o filho recebem todos os cuidados assistenciais. $\mathrm{O}$ recém-nascido logo após o nascimento permanece com a mãe 24 horas por dia até a alta hospitalar que se dá o nome de alojamento conjunto, tendo como vantagens favorecer o aleitamento materno em livre demanda, fortalecer o vínculo e favorecer a oportunidade em que o enfermeiro proporciona orientações à puérpera por meio de práticas dos cuidados durante a hospitalização (ODININO; GUIRARDELLO, 2010).

No alojamento conjunto, a amamentação é um processo livre e estimulado pela equipe de assistência. Porém, muitas mães se encontram com dificuldades durante esse processo, o que pode acarretar a um desmame precoce. Portanto, o profissional de saúde deve estar atento ao aconselhamento sobre a importância do aleitamento materno e a técnica no manejo da amamentação. $\mathrm{O}$ aconselhamento em amamentação começa no pré-natal e estende até o período de lactação, assim, contribuindo para a saúde do binômio mãe-filho (CAMINHA et al., 2008).

Os cuidados de enfermagem estão subdivididos em cuidados diretos (básicos e específicos) e indiretos. Dentre os cuidados diretos, são realizados cuidados básicos àqueles 
recém-nascidos independentemente de alguma complicação: higiene corporal; cuidado com o coto umbilical; verificação de sinais vitais e peso; alimentação e hidratação; observação das eliminações e manter o bebê aquecido em contato com a pele da mãe, já a realização de cuidados específicos são diretamente associados ao RN com alguma complicação: isolamento; complicações do trato gastrointestinal e do tegumento; prematuridade; infecções, dentre outras. Os cuidados indiretos são aqueles relacionados com o ambiente do berçário, materiais, equipamentos e pessoal (OLIVEIRA; RODRIGUES, 2005).

O papel desempenhado pelo enfermeiro dentro de uma maternidade é primordial, estimulando a aproximação entre a mãe e o bebê, realizando os cuidados imediatos, de modo que essa conduta seja observada como um elemento que propicia a aproximação dessa interação, favorecendo o vínculo mãe e filho (CASTELLO; MORAES, 2009).

Considerando a importância das atividades desenvolvidas pelo enfermeiro e os cuidados oferecidos à mãe e ao bebê, cada dia estuda-se a possibilidade de que o vínculo entre ambos é verdadeiro e é dever dos profissionais de saúde aconselhar as mães sobre os cuidados básicos para estimular este vínculo. Dessa forma, nesse trabalho será relevante avaliarmos a percepção das gestantes ao reconhecer a importância da atuação dos enfermeiros frente aos cuidados prestados, fortalecendo o vinculo entre mãe-bebê.

Este trabalho teve como objetivo identificar na literatura a percepção da gestante frente a atuação do enfermeiro nos primeiros cuidados com RN. Descrever a percepção da gestante que fortaleceu o vinculo mãe e filho. Verificar a interferência da atuação do enfermeiro na aproximação entre o binômio e compreender como o enfermeiro contribui para facilitar a aproximação entre mãe-bebê.

\section{METODOLOGIA}

Trata-se de um estudo de revisão integrativa, de aspecto quantitativo, relacionado à percepção da gestante na atuação do enfermeiro, fortalecendo o vinculo mãe-bebê.

Foram utilizados como descritores: percepção das gestantes, cuidados de enfermagem, vínculo entre mãe e filho, recém-nascido, além de estudos que abordem a atuação de enfermagem materno-infantil. Os períodos de busca dos periódicos foram de setembro a outubro de 2014, através da base de dados online Scielo e Biblioteca Virtual em Saúde, todos os artigos completos.

Para a análise dos dados selecionados, foram utilizados os seguintes critérios de inclusão: artigos em periódicos entre o período de 2005 a junho de 2013. Foram analisados os artigos que incluíssem a temática da percepção das gestantes na atuação do enfermeiro, 
1 fortalecendo o vinculo mãe-bebê. Como critérios de exclusão: periódicos que não relacionavam com o tema e publicações em outros idiomas.

Ao final, a amostra foi composta por 12 artigos científicos. Após a análise, os artigos selecionados seguiram dois eixos temáticos: sentimentos das mulheres em relação mãe e filho e percepção das mulheres em relação aos profissionais. O resultado foi apresentado a partir das informações dispostas em forma de quadros, a fim de analisar, discutir, comparar os dados encontrados e assim, elaborar as discussões e considerações finais.

\section{RESULTADOS E DISCUSSÃO}

Os artigos em estudo foram publicados em periódicos em diferentes áreas temáticas, sendo que predomina 07 periódicos na área de enfermagem (Revista de Enfermagem UFPE; Esc. Anna Nery Revista Enfermagem; Revista Brasileira de Enfermagem-REBEn; Revista de Enfermagem UERJ; Rev. Esc. Enferm. USP; Texto Contexto de Enfermagem), 04 na área de psicologia (Estudos de Psicologia; Contemporânea-Psicanálise e transdisciplinaridade; Psicologia: Teoria e Pesquisa; Revista IGT na rede) e 01 na área de ciências e saúde (Revista CEFAC).

Os descritores mais empregados pelos autores foram parto, presente em 06 artigos, seguidos de relações afetivas e cuidados de enfermagem em 05, aleitamento materno, pré-natal e maternidade em 02 artigos.

O conteúdo dos artigos que foram dispostos na Tabela 1 abordam os objetivos, metodologias e população de estudo. Conforme apresentado na Tabela 1, constatou-se que os títulos dos artigos designou conteúdos que envolviam as relações afetivas entre mãe e o bebê, percepções e sentimentos das gestantes e contribuição do enfermeiro através dos cuidados com o RN.

Com relação aos objetivos, esses estiveram relacionados, de um modo geral, a percepção das gestantes com os cuidados de enfermagem, como o enfermeiro contribui com as relações afetivas entre mãe e filho e aleitamento materno.

Em relação ao delineamento da pesquisa, 03 artigos são de revisão de literatura, 03 artigos de estudo quali e quantitativo, 02 de estudos descritivo qualitativo, 01 de estudo quantitativo, 01 de estudo descritivo, 01 de estudo transversal descritivo e 01 de estudo qualitativo exploratório descritivo.

Tabela 1 - Classificação dos artigos examinados segundo autores, ano de publicação, objetivos, metodologia e população estudada. Período 2005 a 2013. 


\begin{tabular}{|c|c|c|c|}
\hline $\begin{array}{l}\text { THOMAZ, A.C.P. } \\
\text { et al., } 2005 .\end{array}$ & $\begin{array}{l}\text { Analisar as primeiras relações afetivas } \\
\text { entre mães e verificar as diferenças na } \\
\text { relação afetiva entre eles e a importância } \\
\text { do contato físico para a formação dessa } \\
\text { primeira relação. }\end{array}$ & $\begin{array}{l}\text { Estudo quali e } \\
\text { quantitativo }\end{array}$ & $\begin{array}{l}28 \text { puérperas } \\
\text { e seus recém- } \\
\text { nascidos. }\end{array}$ \\
\hline BORSA, J.C, 2006. & $\begin{array}{l}\text { Promover reflexões a respeito da } \\
\text { importância da figura materna e da } \\
\text { formação do vinculo mãe-bebê nos } \\
\text { primeiros momentos da vida da criança. }\end{array}$ & $\begin{array}{l}\text { Estudo } \\
\text { revisão }\end{array}$ & $\begin{array}{l}\text { Mulheres em } \\
\text { período } \\
\text { gestacional }\end{array}$ \\
\hline $\begin{array}{l}\text { SILVA, L.R; et al., } \\
2006 .\end{array}$ & $\begin{array}{l}\text { Apresentar uma revisão de literatura dos } \\
\text { fatores e características da interação } \\
\text { mãe-filho a luz da psicologia do } \\
\text { desenvolvimento. }\end{array}$ & $\begin{array}{l}\text { Estudo } \\
\text { revisão de } \\
\text { literatura }\end{array}$ & $\begin{array}{l}\text { Díade mãe- } \\
\text { filho }\end{array}$ \\
\hline $\begin{array}{l}\text { CRUZ,D. C. S; } \\
\text { SUMAN, N. S; } \\
\text { SPINDOLA, T, } \\
2007 .\end{array}$ & $\begin{array}{l}\text { Compreender como o momento do parto } \\
\text { dói percebido pelas mães e de que } \\
\text { maneira os profissionais contribuíram } \\
\text { para facilitar sua aproximação ao recém- } \\
\text { nascido. }\end{array}$ & $\begin{array}{l}\text { Estudo } \\
\text { descritivo } \\
\text { qualitativo. }\end{array}$ & 25 multíparas \\
\hline $\begin{array}{l}\text { MARQUES, M. C. } \\
\text { S; MELO, A. M, } \\
2008 .\end{array}$ & $\begin{array}{l}\text { Avaliar a amamentação em bebês do } \\
\text { alojamento conjunto. Identificar e avaliar } \\
\text { as dificuldades no inicio do aleitamento } \\
\text { materno. }\end{array}$ & $\begin{array}{l}\text { Estudo } \\
\text { transversal } \\
\text { descritivo. }\end{array}$ & $\begin{array}{l}100 \text { binômios } \\
\text { mães e } \\
\text { recém- } \\
\text { nascidos. }\end{array}$ \\
\hline $\begin{array}{l}\text { CASTELLO, L. N; } \\
\text { MORAES, K. F. B, } \\
2009 .\end{array}$ & $\begin{array}{l}\text { Revelar como as adolescentes grávidas } \\
\text { estabelecem e vivenciam a construção do } \\
\text { contato afetivo com o feto no período } \\
\text { gestacional. }\end{array}$ & $\begin{array}{l}\text { Estudo quali e } \\
\text { quantitativo }\end{array}$ & $\begin{array}{l}5 \\
\text { adolescentes } \\
\text { entre } 14 \text { e } 19 \\
\text { anos e } \\
\text { primíperas. }\end{array}$ \\
\hline $\begin{array}{l}\text { MATOS, T. A; et } \\
\text { al., } 2010 .\end{array}$ & $\begin{array}{l}\text { Compreender o significado do contato } \\
\text { precoce pele-a-pele mãe-filho. Identificar } \\
\text { características do estabelecimento desse } \\
\text { contato e contribuição da enfermagem. }\end{array}$ & $\begin{array}{l}\text { Estudo quali e } \\
\text { quantitativo }\end{array}$ & 9 mães. \\
\hline $\begin{array}{l}\text { ODININO, N. G; } \\
\text { GUIRARDELO, E. } \\
\text { B, } 2010 .\end{array}$ & $\begin{array}{l}\text { Avaliar a satisfação das puérperas com } \\
\text { os cuidados e enfermagem recebidos em } \\
\text { um alojamento conjunto. Verificar se a } \\
\text { mesma difere com relação a alguma } \\
\text { variáveis demográficas e obstétricas. }\end{array}$ & $\begin{array}{l}\text { Estudo } \\
\text { descritivo }\end{array}$ & $\begin{array}{l}187 \\
\text { puérperas }\end{array}$ \\
\hline $\begin{array}{l}\text { ROSA, R; et al, } \\
2010 .\end{array}$ & $\begin{array}{l}\text { Identificar e analisar os sentimentos } \\
\text { maternos expressados pelas mães durante } \\
\text { o contato intimo com os filhos logo após } \\
\text { o parto. }\end{array}$ & $\begin{array}{l}\text { Estudo } \\
\text { qualitativo } \\
\text { exploratório } \\
\text { descritivo. }\end{array}$ & $\begin{array}{l}11 \text { mulheres } \\
\text { e seus filhos. }\end{array}$ \\
\hline $\begin{array}{l}\text { PICCININI, C.A; et } \\
\text { al., } 2012 .\end{array}$ & $\begin{array}{l}\text { Investigar as percepções e sentimentos } \\
\text { de gestantes sobre a assistência pré-natal } \\
\text { e se as demandas emocionais eram } \\
\text { consideradas no atendimento. }\end{array}$ & $\begin{array}{l}\text { Estudo } \\
\text { quantitativo. }\end{array}$ & $\begin{array}{l}36 \text { gestantes } \\
\text { de } 20 \text { a } 35 \\
\text { anos. }\end{array}$ \\
\hline $\begin{array}{l}\text { VELHO, M.B; et } \\
\text { al., } 2012 .\end{array}$ & $\begin{array}{l}\text { Identificar a contribuição das pesquisas } \\
\text { desenvolvidas em âmbito nacional e } \\
\text { internacional, sobre a percepção do parto } \\
\text { normal e Cesário pelas mulheres que os }\end{array}$ & $\begin{array}{l}\text { Revisão } \\
\text { integrativa. }\end{array}$ & $\begin{array}{l}\text { Mulheres que } \\
\text { realizaram } \\
\text { parto normal } \\
\text { e Cesário }\end{array}$ \\
\hline
\end{tabular}


BARRETO, C. N; Conhecer o significado do pré-natal para et al., 2013. gestante de um psf.

Estudo

descritivo

qualitativo.
12 mulheres que estavam realizando o pré-natal.
1

Durante a analise dos artigos estudados foram encontrados sentimentos positivos e negativos das mulheres em relação mãe-filho (Tabela 2). Foram encontrados nos sentimentos negativos: preocupação; medo da separação do bebê; sensação de afastamento e dificuldades iniciais na amamentação. Os sentimentos positivos foram: cuidado essencial do pré-natal (formação de vínculo); redução das preocupações; maior vinculo mãe-bebê; contato precoce; alegria, felicidade e realização, relacionadas ao parto normal e Cesário e que os primeiros contatos propiciam o reconhecimento entre mãe/filho.

\section{Tabela 2 - Sentimentos das mulheres em relação mãe-filho}

\begin{tabular}{|c|c|c|}
\hline Sentimento negativo & $\mathrm{N}^{\circ}$ de artigo & Referência \\
\hline $\begin{array}{l}\text { 1.Preocupação, medo da } \\
\text { separação do bebê, sensação } \\
\text { de afastamento. }\end{array}$ & 3 & $\begin{array}{l}\text { CRUZ, 2007; ROSA et al., } \\
\text { 2010; VELHO et al., } 2012 .\end{array}$ \\
\hline $\begin{array}{l}\text { 2.Dificuldades iniciais na } \\
\text { amamentação. }\end{array}$ & 1 & MARQUES; MELO, 2008. \\
\hline Sentimento positivo & $\mathrm{N}^{\circ}$ de artigo & Referência \\
\hline $\begin{array}{l}\text { 1.Cuidado essencial do pré- } \\
\text { natal (formação de vinculo). }\end{array}$ & 2 & $\begin{array}{l}\text { BARRETO et al.,2013; } \\
\text { PICCININI et al., } 2012 .\end{array}$ \\
\hline $\begin{array}{l}\text { 2.Reduções das } \\
\text { preocupações, maiores } \\
\text { vinculam mãe-bebê (Contato } \\
\text { afetivo). }\end{array}$ & 3 & $\begin{array}{l}\text { PICCININI et al., } \\
\text { 2012;CASTELLO et al., } \\
\text { 2009;BORSA, } 2006 .\end{array}$ \\
\hline 3.Contato precoce. & 3 & MATOS et al., 2010; \\
\hline $\begin{array}{l}\text { 4.Alegria, felicidade } \\
\text { realização, relacionadas ao } \\
\text { parto normal e Cesário. }\end{array}$ & 2 & $\begin{array}{l}\text { THOMAZ et al., 2005; } \\
\text { BORSA, 2006. } \\
\text { VELHO et al., 2012; } \\
\text { CASTELLO, L. N; } \\
\text { MORAES, K. F. B, } 2009 .\end{array}$ \\
\hline $\begin{array}{l}\text { 5.Os primeiros contatos } \\
\text { propiciam o reconhecimento } \\
\text { entre mãe/filho. }\end{array}$ & 3 & $\begin{array}{l}\text { ROSA et al., 2010; } \\
\text { THOMAZ, A.C.P. et al., } \\
\text { 2005; CASTELLO, L. N; } \\
\text { MORAES, K. F. B, } 2009 .\end{array}$ \\
\hline
\end{tabular}

Na Tabela 3, foi descrito a percepção das mulheres em relação aos profissionais de enfermagem. Foram encontrados que a contribuição de enfermagem no contato precoce pele a pele é significativa; satisfação do cuidado profissional e profissional como fonte de apoio. 
2 Tabela 3 - Percepção das mulheres em relação ao profissional de enfermagem

\begin{tabular}{lcl}
\hline \multicolumn{1}{c}{ Percepção } & $\mathbf{N}^{\mathbf{0}}$ de artigo & \multicolumn{1}{c}{ Referências } \\
\hline $\begin{array}{l}\text { 1. A contribuição de enfermagem no } \\
\text { contato precoce pele a pele é } \\
\text { significativa. }\end{array}$ & 2 & $\begin{array}{l}\text { MATOS et al, 2010; CRUZ, } \\
\text { D.C.S; SUMAN, N.S; } \\
\text { SPINDOLA, T, 2007. }\end{array}$ \\
$\begin{array}{l}\text { 2. Satisfação do cuidado profissional. } \\
\text { ODININO;GUIRARDELLO, } \\
\text { 3. Profissional como fonte de apoio. }\end{array}$ & $2010 ;$ VELHO et al., 2012. \\
& $\begin{array}{l}\text { PICCININI et al., 2012. } \\
\text { SILVA et al., 2006; } \\
\text { BARRETO, C.N; et al., 2013. }\end{array}$ \\
\hline
\end{tabular}

3

4

5

6

7

8

9

10

Dentre os sentimentos negativos foi revelado que os cuidados prestados ao bebê, distante do olhar materno, geram nas mães preocupação, sensação de afastamento e medo da separação do bebê (CRUZ et al., 2007).

Entre um dos sentimentos negativos, destacando-se as dificuldades no inicio do aleitamento materno, presente em dois artigos. Essas dificuldades encontradas foram: adequação da sucção, má posição corporal da mãe e do bebê durante a mamada, o aspecto da anatomia das mamas e afetividade. O enfermeiro como cuidador deve oferecer a mãe um ambiente tranquilo, auxilia-la ao posicionamento mais confortável para ela e para a criança, o bebê tem que abocanhar toda a auréola da mama, os lábios devem estar posicionados para o lado de fora, a mãe deve favorecer confiança para o filho e evitar manobras que apressem o bebê no aleitamento (MATOS et al., 2010).

Em relação aos sentimentos positivos as mulheres compreenderam que o pré-natal e suas ações, devido a atenção e a formação de vínculo, influenciam na evolução da gestação, na redução das preocupações sobre a própria saúde e a do bebê e no vinculo mãe-bebê (BARRETO et al., 2013).

Quanto a percepção das mulheres em relação aos profissionais de enfermagem, fica evidente que os primeiros contatos são preponderante para propiciar o reconhecimento entre mãe e filho, sendo que a contribuição de enfermagem no estabelecimento desse contato é significativa ( ROSA et al., 2010).

De acordo com os resultados encontrados percebeu-se que muitos descritores não foram coerentes com o texto, para melhor localização das palavras chaves é importante que os autores façam pesquisa definindo as palavras chaves que melhor se encaixa no conteúdo de estudo, 
1 encontra-se disponível na base de dados denominada DeCS - Descritores em Ciência da Saúde 2 e/ou MeSH - Medical Subject Headings, conduzindo a pesquisa mais eficiente, associando com o objetivo da pesquisa (CASTRO, 2001).

4 Nos artigos o papel do enfermeiro como cuidadores da saúde e seus benefícios é 5 fundamental para o vinculo entre mãe e filho. Segundo Piccinini (2012), no pré-natal o 6 enfermeiro como cuidador é responsável por suprir as necessidades emocionais da mãe, além 7 da saúde física da gestante e do bebê antes do nascimento. Após o nascimento segundo Odinino 8 e Guirardelo (2010), o enfermeiro proporciona a mãe durante a hospitalização o aleitamento 9 materno em livre demanda e orientações a puérpera através de praticas de cuidados.

Com relação aos sentimentos das mulheres foi possível verificar que as mulheres relataram satisfação com os cuidados de enfermagem, sendo os sentimentos positivos. Embora foi relatado sentimentos negativos relacionados aos domínios de educação e confiança. De acordo com Cruz (2007), existem outros sentimentos negativos que são preocupação, medo da separação do bebê e sensação de afastamento. Ter esses sentimentos é natural, pois a mãe passa por diversas mudanças, seja elas psicológicas, sentimental e física. Seria relevante os autores descrever como evitar esses sentimentos negativos, e como auxiliar a mãe a lidar com eles.

A respeito às contribuições de enfermagem, segundo os autores as mulheres consideram os cuidados eficaz. A importância da presença da equipe de enfermagem no momento em que a mulher passa a ser mãe e nutriz é fundamental, pois proporciona o inicio do contato e auxilia neste primeiro reconhecimento e fortalecimento entre mãe e filho (MATOS et al., 2010).

\section{CONCLUSÃO}

A realização deste estudo possibilitou pesquisar, compreender e identificar o significado da contribuição da equipe de enfermagem para a formação do vinculo entre mãe e filho. Identificando os sentimentos e as percepções das mulheres em relação aos cuidados prestados pelo enfermeiro, propiciando intervenções na interação com o binômio, visando no reconhecimento mãe-filho que possui evidente importância materna e auxiliando a mãe através do pré-natal, passando total confiança a mesma. 


\section{REFERÊNCIAS BIBLIOGRÁFICAS}

(1)

BARRETO, C. N.; RESSEL, L. B.; SANTOS, C. C.; WILHELM, L. A.; SILVA, S. C.; ALVES, C. N. Atenção pré-natal na voz das gestantes. Revista de enfermagem UFPE on line., Recife, v. 7, n. 5, p. 4354-63, jun. 2013.

BORSA, J. C. Considerações acerca da relação Mãe-Bebê da Gestação ao Puerpério.

Contemporânea - Psicanálise e Transdisciplinaridade, Porto Alegre, n. 2, p. 310-321, abr/mai/jun. 2007.

CASTELLO, L. N.; MORAES, K. F. B. O estabelecimento de contato afetivo durante a gestação, sob a perspectiva da Gestalt-Terapia. Revista IGT na Rede, v. 6, n. 10, p. 145-169, 2009.

CASTRO, E. Terminologia, palavras-chave, descritores em saúde: qual a sua utilidade?. Jornal Brasileiro de AIDS, v. 2, n.1, p. 51-61, 2001.

CRUZ, D. C. S.; SUMAM, N. S.; SPÍNDOLA, T. Os cuidados imediatos prestados ao recémnascido e a promoção do vinculo mãe-bebê. Rev. Esc. Enferm. USP., Rio de Janeiro. v. 41, n. 4, p.690-7, 2007.

MARQUES, M. C. S.; MELO, A. M. Amamentação no alojamento conjunto. Rev. CEFAC, São Paulo. v. 10, n. 2, p.261-271, 2008.

MATOS, T. A.; SOUZA, M. S.; SANTOS, E. K. A.; VELHO, M. B.; SELBERT, E. R. C.; MARTINS, N. M. Contato precoce pele a pele mãe e filho: significado para as mães e contribuições para a enfermagem. Revista Brasileira de Enfermagem., Brasilia. V. 63, n. 6, p 998-1004, 2010.

ODININO, N. G.; GUIRARDELLO, E. B. Satisfação da Puérpera com os cuidados de Enfermagem recebidos em um alojamento conjunto. Texto Contexto Enferm., Florianópolis, v. 19 , n. 4 , p. 682-90, out.-dez. 2010.

PICCININI, C. A.; CARVALHO, F. T.; OURIQUE, L. R.; LOPES, R. S. Percepções e Sentimentos sobre o Pré-natal. Psicologia: Teoria e Pesquisa, Porto Alegre, v. 28, n. 1, p. 27-33, jan.-mar. 2012. 
1 ROSA, R.; MARTINS, F. E.; GASPERI, B. L.; MONTICELLI, M.; SIEBERT, E. R. C.;

2 MARTINS, N. M. Mãe e Filho: Os primeiros laços de Aproximação. Esc. Anna Nery Rev.

3 Enferm., Santa Catarina, v. 14, n. 1, p. 105-12, jan.-mar. 2010.

4

5

6
SILVA, L. R.; CHRISTOFFEL, M. M.; FERNÁNDEZ, A. M.; SANTOS, I. M. M. A Importância da Interação Mãe-Bebê no Desenvolvimento Infantil: A Atuação da Enfermagem Materno-Infantil. Rev. Enferm. UERJ, Rio de Janeiro, v. 14, n. 4, p. 606-12, out.-dez. 2006.

THOMAZ, A. C. P.; LIMA, M. R. T.; TAVARES, C. H. F.; OLIVEIRA, C. G. Relações afetivas entre mães e recém-nascidos a termo e pré-termo: variáveis sociais e perinatais. Estudos de Psicologia, Alagoas, v. 10, n. 1, p. 139-146. 2005.

VELHO, M. B.; SANTOS, E. K. A.; BRÜGGEMANN, O. M.; CAMARGO, B. V. Vivência do Parto Normal ou Cesário: Revisão Integrativa sobre a Percepção de Mulheres. Texto Contexto Enferm., Florianópolis, v. 21, n. 2, p. 458-66. 2012. 